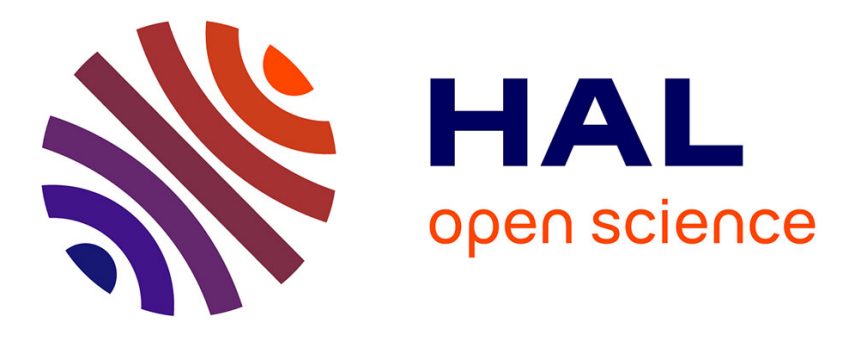

\title{
Simultaneous Displacement/Force Self-Sensing in Piezoelectric Actuators and Applications to Robust Control.
}

Micky Rakotondrabe, Ioan Alexandru Ivan, Sofiane Khadraoui, Philippe Lutz, Nicolas Chaillet

\section{To cite this version:}

Micky Rakotondrabe, Ioan Alexandru Ivan, Sofiane Khadraoui, Philippe Lutz, Nicolas Chaillet. Simultaneous Displacement/Force Self-Sensing in Piezoelectric Actuators and Applications to Robust Control.. IEEE/ASME Transactions on Mechatronics, 2015, 20 (2), pp.519-531. hal-01303384

\section{HAL Id: hal-01303384 \\ https://hal.science/hal-01303384}

Submitted on 28 Apr 2016

HAL is a multi-disciplinary open access archive for the deposit and dissemination of scientific research documents, whether they are published or not. The documents may come from teaching and research institutions in France or abroad, or from public or private research centers.
L'archive ouverte pluridisciplinaire HAL, est destinée au dépôt et à la diffusion de documents scientifiques de niveau recherche, publiés ou non, émanant des établissements d'enseignement et de recherche français ou étrangers, des laboratoires publics ou privés. 


\title{
Simultaneous displacement/force self-sensing in piezoelectric actuators and applications to robust control
}

\author{
Micky RAKotondrabe ${ }^{1 *}$, member, IEEE, Ioan Alexandru IVAN ${ }^{1-2}$, member, IEEE, Sofiane KhadRAOUi ${ }^{1}$, \\ Philippe LUTZ ${ }^{1}$, member, IEEE and Nicolas CHAILLET ${ }^{1}$, member, IEEE
}

\begin{abstract}
Self-sensing technique consists in using an actuator as a sensor at the same time. This is possible for most of actuators with physically reversible principle such as piezoelectric materials. The main advantages of self-sensing are: 1) the embeddability of the measurement technique, and 2) its low cost as no additional sensor is required.

This paper presents a self-sensing technique for piezoelectric actuators used in precise positioning applications like micromanipulation and microassembly. The main novelty is that both displacement and force signals can be simultaneously estimated. This allows a feedback control using one of these two signals with a display of the other signal. To demonstrate this advantage, a robust $H_{\infty}$ feedback control on displacement with real-time display of the force is used as an application of the proposed selfsensing technique. Along the paper, experimental results obtained with a piezoelectric cantilever actuator validate and demonstrate the efficiency of the proposed self-sensing.
\end{abstract}

Index Terms-Self-sensing, piezoelectric actuator, displacement and force estimation, robust $H_{\infty}$ control.

\section{INTRODUCTION}

$\mathbf{M}$ Icropositioning (or precise positioning) tasks, the basis of microassembly and micromanipulation applications, require a very high accuracy which is in the order of micrometer or even submicrometer. To reach such severe performances, the used microrobots, micromanipulators and actuated microsystems are often based on active and smart materials instead of hinges and joint systems which generate imprecision due to the mechanical clearances. Among the used active materials, piezoelectric materials are the most recognized. They have been successfully used to develop stepper microrobots [1] [2], Atomic Force Microscopes (AFM) [3] and continuous microactuators such as piezocantilevers and microgrippers 4 [5]. This recognition is mainly thanks to the high resolution (at the nanometer level), the high bandwidth (more than the $\mathrm{kHz}$ ) and the relatively high force density that they offer. For all that, piezoelectric materials (in particular piezoceramics) exhibit hysteresis and creep nonlinearities. These nonlinearities become non-negligible when the applied electrical field -and therefore the range of deformation- are large. Hysteresis and

1: FEMTO-ST Institute, UMR CNRS 6174 - UFC / ENSMM / UTBM Automatic Control and Micro-Mechatronic Systems depart., AS2M

24, rue Alain Savary

25000 Besançon - France

2: Valahia University of Targoviste

FIE/ICSTM, B-dul Unirii 18-20, 130082 Targoviste, Romania

*: Corresponding author: mrakoton@ femto-st.fr

Paper type: regular paper. creep limit the accuracy of the developed actuators. In order to enhance the performances, several works have been devoted to the modeling and to the control of piezoelectric actuators within these ten last years. These works include closed-loop control techniques [6]- [9], open-loop control techniques [10]- [17] and the combination of both [20].

In feedback techniques, basic control schemes like PIDstructure and more advanced control laws $\left(H_{\infty}\right.$, passivity, interval, nonlinear control, etc.) have been successfully used. The main advantage of feedback is the robustness: 1) the specifications are satisfied in almost all the cases, and 2) the effects of model uncertainties and of the external disturbances can be rejected efficiently. However, feedback requires sensors. This constitutes the main limitation of feedback techniques at the micro/nano world and related applications. On the one hand, sensors that have the required performances (accuracy, bandwidth and low noises) are bulky and expensive: for instance triangular optical sensors, interferometers, etc. On the other hand, sensors that are packageable and cheap are fragile, difficult to handle and noisy: for instance strain gages. Further, feedforward techniques have been successfully introduced to bypass this limitation as they do not need sensors. They have the particularity of yielding really packaged controlled systems. Nevertheless, feedforward techniques fail with uncertain models and in presence of external disturbances. To resume, finding the compromise between satisfying the desired performances and satisfying the available space is difficult.

Promising approaches avoiding external sensors in meso and micro systems are the so-called self-sensing methods. They allow both actuation and sensing capabilites, and are especially (but not necessarily) used for piezoelectric materials based actuators and systems. Given the reversibility of the piezoelectric effect, self-sensing does or should not influence the actuation capabilities in terms of range or dynamics. It could provide simultaneous quantitative information of end-effector displacement, manipulation force, close contact detection or even temperature evaluation. If well designed, self-sensing approaches could combine the advantages from feedback (robustness) and from feedforward (packageability) for piezoelectric actuators.

The first use of "self-sensing" term goes back to 1992 when J.J. Dosch et al 21] successfully damped the vibration of a piezoelectric beam without using the aid of external sensors. In their works, the voltage drop provided from a capacitive bridge was processed in an analog circuit, amplified and returned back 
to the piezoelectric element. In that time, several independent applications began to emerge for beam vibration control or stack piezo devices for micropositioning. Several years later T. Taigami et al [22] applied the method for force selfsensing and for the control of a large size bimorph actuator by using a half-bridge circuit, a voltage follower and a computerbased data acquisition system. The authors experimentally verified that the stiffness of the grasping object does not affect the measurement; however electronic schematic limited the applied voltage range and nonlinearities (hysteresis and creep) were not compensated. Since, self-sensing is widely used for vibration control of flexible structures [23] [24]. All these existing self-sensing works usually offered shortterm measurements: measurement is not available for no more than 5 seconds in general. Moreover, very few works refer to the use of self-sensing method for the control of positioning. Consequently, these existing works could not be applied to measure signals (displacement and force) and to control systems in micropositioning tasks such as micromanipulation or microassembly. Indeed, such applications require the measurement of displacement/force and the combination of dynamic and static sensing/measurement with duration from several tens of seconds to several minutes. This duration corresponds to the transport of the manipulated micro-objects where the displacement has to be controlled precisely and the manipulated force to be known. Newly, in our previous works

[25], a new scheme of self-sensing has been proposed for precise measurement of the displacement in its steady-state for more than $600 \mathrm{~s}$. Further we have extended the proposed scheme to include the measurement of steady-state force signal [26]. But as the proposed scheme was for quasi-static selfsensing, it could not be used for control application. This paper aims to develop a scheme for static and dynamic selfsensing in order to allow the control of piezoelectric actuators used in micropositioning tasks. The proposed approach provides a measurement and estimation of the displacement in both steady-state and dynamics enabling the positioning control for long term period. Furthermore, the scheme also allows the measurement and estimation of the steady-state force at the same time which is very useful for the user to overlook the manipulation force applied to the object. Finally, to demonstrate these advantages, we employ the proposed selfsensing to a feedback control application. Experimental tests were carried to validate the self-sensing techniques and to validate its applicability to control applications. They clearly demonstrate the efficiency of the proposed self-sensing.

The paper is organized as follows. In section-II we remind the quasi-static self-sensing as in $\llbracket 25 \rrbracket$ and $\llbracket 26$. In section-III, we present the proposed self-sensing allowing the steady-state and dynamic displacement measurement and the steady-state force measurement at the same time. Finally, section-IV is devoted to a control application of micropositioning tasks.

\section{REMIND OF THE QUASI-STATIC DISPLACEMENT AND FORCE SELF-SENSING}

\section{A. The framework of micromanipulation and microassembly}

Within the framework of micromanipulation and microassembly applications, microgrippers based on piezoelec- tric actuators with cantilever structure are used. In general two actuators are employed to constitute a microgripper. Each actuator has a rectangular section and is usually unimorph. Bimorph actuators are rarely used because of the difficulty to fabricate them and to setup the electrical connection. A unimorph piezoelectric actuator is composed of two layers glued themselves: a piezoelectric layer (often based on lead zirconate titanate (PZT) material) and a passive layer (often based on copper or nickel materials) (Fig. 1. - a). When a voltage $U$ is applied to the piezoelectric layer, it contracts or expands following the converse principle of piezoelectric effect. As a result, the whole cantilever bends (Fig. 1.b). The microgripper is able to position very precisely (i.e. to pick, transport and place very precisely) a small object if each actuator is conveniently controlled [5] (Fig. 1 1 c). An essential feature of self-sensing applied to microgripper's actuators is the ability to simultaneous measure and estimate the displacement at the tip and the force applied to the object (Fig. 1.d). This is what this paper claims to propose.

\section{B. Quasi-static displacement self-sensing}

Self-sensing, i.e. the use of an actuator as also a sensor, is possible thanks to the reversibility of piezoelectric effect. Its principle scheme is pictured in Fig. 2.a $\mid 25$. When a voltage $U$ is applied to the actuator, a bending $y$ is obtained (actuation). During this bending, an electrical charge $Q$ appears on the electrodes of the actuator. This charge can be amplified by a convenient electrical circuit and transformed into an exploitable voltage $U_{o}$. From the available signals $U$ and $U_{o}$, an observer calculated from the electrical circuit and from the model of the piezoelectric actuator provides an estimate $\hat{y}_{s}$ of the displacement $y$ at its steady-state. Both the electrical circuit and the observer constitute the sensing/measurement part of the self-sensing.

Fig. 2.b pictures the proposed electrical circuit. In order to explain its principle, the equivalent electrical circuit of the piezoelectric actuator has been introduced. This equivalent circuit is composed of three parallel components: the internal generator which supplies charge $Q_{s}$ due to internal stress of the material when deformed, the internal piezoelectric capacitor $C_{p}$ and the leakage resistor $R_{f p}$. The total charge $Q$ that appears on the electrodes of the actuator is therefore composed of the internal charge $Q_{s}$ (piezoelectric effect) and the charge $Q_{U}=C_{p} U$ (dielectric effect): $Q=Q_{s}+C_{p} U$.

Experiments shown however that $Q_{s}$ is very weak relative to $Q_{U}$. This will drastically decrease the accuracy of a selfsensing measurement as the sensitivity to the internal stress, and therefore to the deformation, is very weak. To reduce this unwanted property, we proposed to put in parallel with the piezoelectric actuator a reference capacitor $C_{r}$ such that $C_{r}=C_{p}$. By inverting the voltage applied to $C_{r}$ relative to the voltage $U$ applied to the actuator, the charge due to the dielectric effect $\left(Q_{U}=C_{p} U\right)$ can be compensated. Consequently, the final charge that will be amplified by the op-amp is: $Q-C_{r} U=Q_{s}+C_{p} U-C_{r} U=Q_{s}$. However, the piezoelectric capacitor varies following the applied voltage $U$ [27], i.e. $C_{p}=C_{p}(U)$. Hence the dielectric effect is not completely compensated by the reference capacitor $C_{r}$. 
Consequently the exploitable charge becomes:

$$
Q-C_{r} U=Q_{s}+C_{p}(U) U-C_{r} U=Q_{s}+\Delta_{Q}(U)
$$

where $\Delta_{Q}(U)$ is residual charge that depends on the applied voltage $U$. If $\Delta_{Q}(U)=0$, the charge due to the dielectric effect was fully compensated and the self-sensing measurement technique would be highly sensitive to the deformation of the piezoelectric material. Although $\Delta_{Q}(U) \neq 0$, it is still much less than $Q_{s}$ making the sensitivity of the technique still high. In Fig. 2. b, the capacitor $C$ is used to amplify the charge while the resistor $R_{d i s c}$ and the relay $k_{d i s c}$ allow resetting the output $U_{o}$ in order to avoid any unexpected saturation issues. Finally, the op-amp is considered to have a very high input impedance. In the sequel, the voltage $U$ is also called input voltage, control voltage or driving voltage. It corresponds to the input control signal of the actuator.

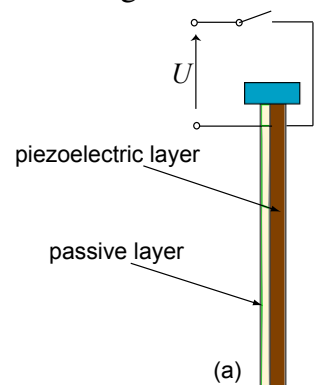

(a)

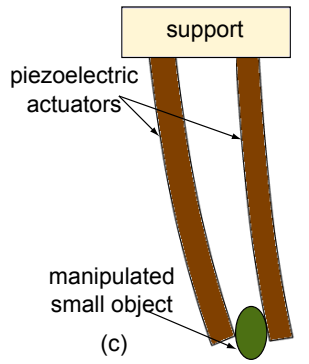

(c)

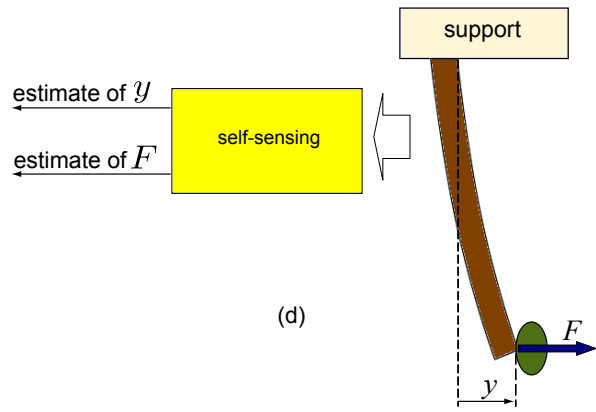

Fig. 1: (a) and (b), principle of a unimorph piezoelectric cantilevered actuator. (b) a microgripper with two piezoelectric cantilevered actuators. (c) simultaneous estimation of the displacement and of the manipulation force by using self-sensing.

Noticing that $Q=\alpha y$, where $\alpha$ is the displacement-charge coefficient, the output and exploitable voltage $U_{o}$ is expressed as follows [25]:

$$
U_{o}=-\frac{1}{C}\left(\alpha y-C_{r} U+\frac{1}{R_{f p}} \int_{0}^{t} U d t+Q_{D A}(t, U)\right)
$$

where $Q_{D A}(t, U)$ is the dielectric absorption of the equivalent capacitor $C_{p}$ in the piezoelectric actuator [28]. Dielectric absorption is the property of a dielectric, like piezoelectric materials, which prevents a capacitor from totally discharging, even when short-circuited for a short period of time. The dielectric absorption can be approximated by a linear model:

$$
Q_{D A}(s)=\frac{k_{D A}}{\left(1+\tau_{D A} s\right)} U(s) \Leftrightarrow Q_{D A}(s)=Q_{t f D A}(s) U(s)
$$

where $k_{D A}$ is the static gain, $\tau_{D A}$ is the constant time and $s$ is the Laplace variable. Expression $Q_{t f D A}(s)=\frac{k_{D A}}{\left(1+\tau_{D A} s\right)}$ is the transfer function of the dielectric absorption.
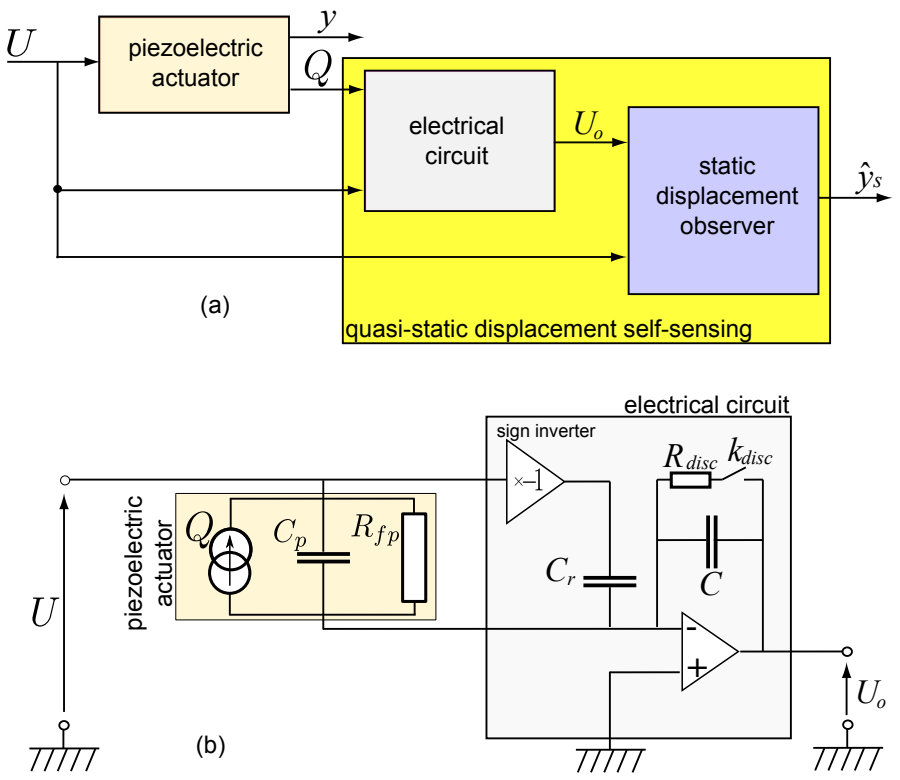

Fig. 2: (a) Principle scheme of the quasi-static displacement self-sensing. (b) the electrical circuit [25].

The observer that gives an estimate $\hat{y}_{s}$ of $y$ at its steady-state is therefore described by (Eq. 4) [25]:

$$
\hat{y}_{s}=\frac{1}{\alpha}\left(\left(C_{r} U-C U_{o}\right)-Q_{D A}(t, U)-\frac{1}{R_{f p}} \int_{0}^{t} U d t\right)
$$

The different parameters are identified following an experimental procedure or computed from the physical and geometrical properties of the piezoelectric actuator and from the parameters of the electrical circuit (see [25]).

\section{Quasi-static displacement and force self-sensing}

The previous self-sensing only provides the (steady-state) displacement information. This is only utilizable when the actuator has a free displacement and thus when no object is manipulated. When the actuator is in contact with an object and consequently when a manipulation force exists, the observer defined by (Eq. 4) of the previous self-sensing is not anymore valuable. In that case, the force and the displacement need to be modelled at the same time. By using an appropriate observer from this model, an observer that estimates the real displacement $y$ and the real force $F$ can again be computed. Then, the displacement and force selfsensing is derived. Fig. 3-a shows the new principle scheme of the quasi-static displacement and force self-sensing [26]. 
We remind that the electrical scheme is still similar than that of the quasi-static displacement self-sensing (see Fig. 2-b).

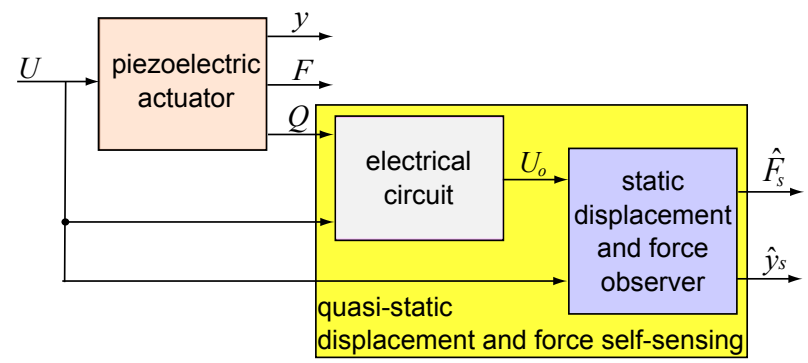

Fig. 3: Principle scheme of the quasi-static displacement and force self-sensing.

The observer that gives an estimate $\hat{y}_{s}$ of the displacement and an estimate $\hat{F}_{s}$ of the force at their steady-state is $\mid 26$ :

$$
\left\{\begin{array}{l}
\hat{F}_{s}=\frac{1}{\beta}\left(\left(C_{r} U-C U_{o}\right)-F_{c r}(t)-F_{h y s}(t)-\frac{1}{R_{f p}} \int_{0}^{t} U d t\right. \\
\hat{y}_{s}^{\text {free }}=\frac{1}{\alpha}\left(\left(C_{r} U-C U_{o}\right)-Q_{D A}(t, U)-\frac{1}{R_{f p}} \int_{0}^{t} U d t\right) \\
\hat{y}_{s}=\hat{y}_{s}^{\text {free }}-s_{p} \hat{F}_{s}
\end{array}\right.
$$

where $\beta$ is the force sensitivity coefficient. It relates the electrical charge appearing on the actuator's surface with the applied external force. $s_{p}$ is the piezoelectric compliance that relates the displacement with the applied external force. Signal $\hat{y}_{s}^{\text {free }}$ corresponds to the estimate steady-state displacement when there is no external force. It corresponds to the estimate displacement presented in (Eq. 4). Finally, signals $F_{c r}(t)$ and $F_{\text {hys }}(t)$ corresponds to the effects of the creep and the hysteresis nonlinearities of the actuator. While the creep is often described by a linear operator, the hysteresis is described by a nonlinear one such as the Bouc-Wen, the Prandtl-Ishlinskii or the Preisach operators. Their compact notations are:

$$
F_{c r}(s)=F_{t f c r}(s) U(s) \quad ; \quad F_{h y s}(s)=\Gamma(U)
$$

with $F_{t f c r}(s)$ a transfer function that captures the creep and $\Gamma(U)$ the nonlinear operator that captures the hysteresis.

\section{COMPLETE DiSPLACEMENT SELF-SENSING WITH STEADY-STATE FORCE ESTIMATION}

The displacement and force self-sensing previously presented is static. It can estimate these signals at their steadystate regime or when working at low frequency, i.e. if $F(t)$ and $y(t)$ are constant we have:

$$
\left\{\begin{array}{l}
\hat{F}_{s}(t)=F(t) \quad \text { for } \quad t \rightarrow \infty \\
\hat{y}_{s}(t)=y(t) \text { for } \quad t \rightarrow \infty
\end{array}\right.
$$

In the Laplace domain, (Eq. 7) is equivalent to:

$$
\left\{\begin{array}{l}
\hat{F}_{s}(s)=F(s) \quad \text { for } \quad s \rightarrow 0 \\
\hat{y}_{s}(s)=y(s) \quad \text { for } \quad s \rightarrow 0
\end{array}\right.
$$

The main advantage of the quasi-static self-sensing is its suitability for micropositioning tasks in micromanipulation and microassembly applications. During these tasks, the object is maintained by the microgripper for several hundreds of seconds with constant force and/or displacement. However, "static measurement" is not proper for feedback control. Dynamic model as well as "dynamic measurement" are required. In this section, we propose a dynamic self-sensing, called complete self-sensing, able to provide steady-state and "dynamic" signals at the same time. More precisely, the proposed selfsensing will be able to provide the steady-state information of the manipulation force and both the steady-state and the "dynamic" information of the displacement which makes the latter signal usable for feedback control.

A. Principle scheme

Fig. 4 gives the principle scheme of the proposed complete self-sensing. It is composed of:

- an electrical circuit that amplifies and transforms the charge $Q$ into an exploitable voltage $U_{o}$. This electrical circuit is similar to that presented in Fig. 2.b.

- a static displacement and force observer that furnishes the steady-state estimates $\hat{y}_{s}$ and $\hat{F}_{s}$. The static observer is described by (Eq. 5).

- and a dynamics observer that furnishes complete information (stead-state and dynamics) $\hat{y}$ on the displacement.

Both the static observer and the dynamics observer constitute the complete observer. The synthesis of the dynamics observer is presented in the next parts.

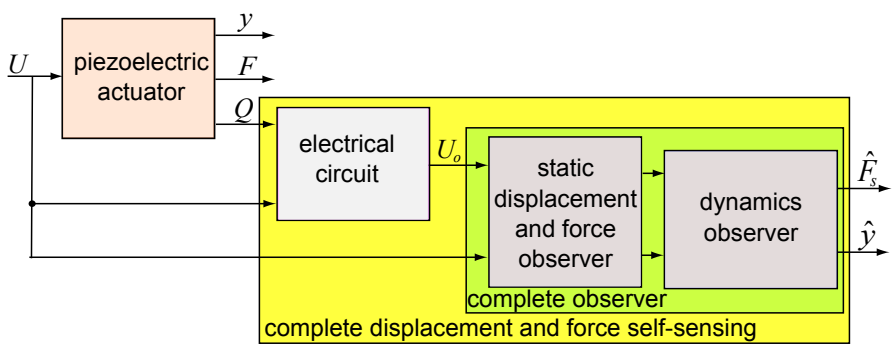

Fig. 4: Principle scheme of the complete displacement and force self-sensing.

\section{B. Conditions for the complete observer}

In order to have a steady-state and dynamics estimation of the displacement, i.e. a full measurement at low and high frequency, the following condition must be respected:

$$
\hat{y}(s)=y(s) ; \quad \forall s \in \mathbb{C}
$$

In the temporal domain, (Eq. 9) is equivalent to:

$$
\hat{y}(t)=y(t) ; \quad \forall t \in \mathbb{R}^{+}
$$

Combining the condition of complete displacement selfsensing in (Eq. 9) and (Eq. 10) with the condition of quasistatic force self-sensing from (Eq. 7) and (Eq. 8), we can write the conditions of complete displacement and force self-sensing as follows:

$$
\begin{aligned}
& \left\{\begin{array}{l}
\hat{F}_{s}(s)=F(s) \quad \text { for } \quad s \rightarrow 0 \\
\hat{y}(s)=y(s) ; \quad \forall s \in \mathbb{R}^{+}
\end{array}\right. \\
& \Leftrightarrow\left\{\begin{array}{l}
\hat{F}_{s}(t)=F(t) \quad \text { for } t \rightarrow \infty \\
\hat{y}(t)=y(t) ; \quad \forall t \in \mathbb{R}^{+}
\end{array}\right.
\end{aligned}
$$




\section{Dynamics observer synthesis}

The model of the piezoelectric actuator that relates the complete information of displacement $y(s)$ with the applied input control $U(s)$ and with the applied steady-state external force $F_{s}(s)$ is (see for eg. $|7|$ ):

$$
y(s)=d_{p} D(s) U(s)-s_{p} F_{s}(s)
$$

where $d_{p}$ is the piezoelectric coefficient and $D(s)$ is the dynamics such that $D(s=0)=1$ (normalization of the dynamics). In this equation, $y=d_{p} D(s) U$ corresponds to the free displacement, i.e. displacement without external force applied. The parameters $d_{p}$ and $D(s)$ can be identified experimentally from the piezoelectric actuator by using a step response method, a harmonic method or a pseudo-random binary sequence methods.

On the other hand, if we consider again the third equation in (Eq. 5):

$$
\hat{y}_{s}(s)=\hat{y}_{s}^{\text {free }}(s)-s_{p} \hat{F}_{s}(s)
$$

we observe that a dynamics we denote $\Delta(s)$ can be introduced in the estimate steady-state model (Eq. 13) in order to have a similar structure than (Eq. 12), and then to have a more complete estimate model:

$$
\hat{y}(s)=\Delta(s) \hat{y}_{s}^{\text {free }}(s)-s_{p} \hat{F}_{s}(s)
$$

Notice that $\Delta(s)$ will certainly be in the dynamics observer. If we find a dynamic observer $\Delta(s)$, the self-sensing will be able to track any transient part of the output displacement that is due to the electrical excitation $U$. However, the transient part of the displacement as response to any applied force $F$ will not be estimated. In this case, only the steady-state value will be tracked. Finally, when both electrical excitation $U$ and mechanical excitation $F$ are applied to the piezoelectric actuator, we have the following results: 1 ) the self-sensing will still track and estimate the transient part and the steady-state value of the output displacement due to the voltage $U, 2$ ) the self-sensing will track and estimate the steady-state only of the displacement due to the force $F$.

The elastic parts in (Eq. 12) and (Eq. 13) being similar, our study can be restricted to the displacement without external force, i.e.:

$$
\left\{\begin{array}{l}
y(s)=d_{p} D(s) U(s) \\
\hat{y}(s)=\Delta(s) \hat{y}_{s}^{\text {free }}(s)
\end{array}\right.
$$

which is equivalent to:

$$
\left\{\begin{array}{l}
\frac{y(s)}{U(s)}=d_{p} D(s) \\
\frac{\hat{y}(s)}{\hat{y}_{s}^{\text {free }}(s)}=\Delta(s)
\end{array}\right.
$$

In order compute the dynamics observer and its gains, we need to write the transfer $\frac{\hat{y}(s)}{U(s)}$ between the estimate complete information $\hat{y}(s)$ and the input control $U(s)$ such that we can compare it with the transfer $\frac{y(s)}{U(s)}$ in (Eq. 16. For that, remind that:

$$
\frac{\hat{y}(s)}{U(s)}=\frac{\hat{y}_{s}^{\text {free }}(s)}{U(s)} \cdot \frac{\hat{y}(s)}{\hat{y}_{s}^{\text {free }}(s)}
$$

where $\frac{\hat{y}(s)}{\hat{y}_{s}^{\text {free }}(s)}=\Delta(s)$ according to (Eq. 16 .

We now need to compute the transfer $\frac{\hat{y}_{s}^{\text {Jree }}(s)}{U(s)}$. To this end, we apply the Laplace transform to (Eq. 4) and use (Eq. 3), we derive:

$$
\hat{y}_{s}^{f r e e}(s)=\frac{1}{\alpha}\left(\left(C_{r}-\frac{1}{\frac{1}{R_{f p}} s}-Q_{t f D A}(s)\right) U-C U_{o}\right)
$$

We know that the relation between the input voltage $U$ and the exploitable voltage $U_{o}$ can be approximated by a linear transfer function $H(s)$ such that [29]:

$$
U_{o}(s)=H(s) U(s)
$$

Replacing $Q_{t f D A}(s)$ by its model in (Eq. 3) and introducing (Eq. 19), (Eq. 18) becomes:

$$
\frac{\hat{y}_{s}^{\text {free }}(s)}{U(s)}=\frac{C_{r}}{\alpha}-\frac{1}{\frac{\alpha}{R_{f p}} s}-\frac{k_{D A}}{\alpha\left(1+\tau_{D A} s\right)}-\frac{1}{\alpha} H(s) ; \quad \forall s \in \mathbb{C}
$$

Based on (Eq. 20), the transfer (Eq. 17) becomes:

$$
\frac{\hat{y}(s)}{U(s)}=\left(\frac{C_{r}}{\alpha}-\frac{1}{\frac{\alpha}{R_{f p}} s}-\frac{k_{D A}}{\alpha\left(1+\tau_{D A} s\right)}-\frac{1}{\alpha} H\right) \frac{\hat{y}(s)}{\hat{y}_{s}^{\text {free }}(s)}
$$

Let us now consider the transfer $\frac{\hat{y}(s)}{\hat{y}_{s}^{f r e e}(s)}=\Delta(s)$ which is in fact the basis of the dynamics observer. Finding this transfer function is a deconvolution problem because its input is a steady-state information while the output is a complete information. Remind that complete information means that it includes the steady-state and the transient parts information. Such problem comes back to the estimation of a transient part after yet reaching a steady-state and is therefore equivalent to a dynamics inversion. The inversion of dynamics have attracted the attention in different applications. In fact, inverting dynamics is delicate in the sense that some conditions should be satisfied [30]. For instance, when using a direct inversion of models, conditions like bi-causality, bi-stability, minimum phase of the models, etc. should be satisfied. Several approaches have therefore been proposed to minimize the number of these conditions which finally limited the usable models. In [31], Fleming and Leang propose the use of an optimal equivalent inverse model as an approximate feedforward dynamics controller (compensator). In our previous works, an inverse multiplicative structure was proposed as inverse model for nonlinearity compensator in piezoelectric actuators [12] 17 and for estimating signal in a compliant platform [32. Inverse multiplicative structure does not require specific conditions, contrary to direct inversion of models. In addition, no computation is required to derive the inverse model. Indeed, as soon as the (direct) model is identified or given, the inverse model is yielded without calculation. This is because the inverse model uses a structure, called inverse multiplicative structure, that contains the initial (direct) model. For these reasons, we propose in this paper to impose the structure of the transfer $\frac{\hat{y}(s)}{\hat{y}_{s}^{\text {free }}(s)}=\Delta(s)$ (the dynamics observer) as inverse multiplicative structure. Let Fig. 5 be the dynamics observer with the proposed structure, where $G_{1}(s), G_{2}(s)$ and $G_{3}(s)$ are the observer gains to be synthesized. As said before, these 
gains are directly based on a direct model and no additional computation is required to determine them.

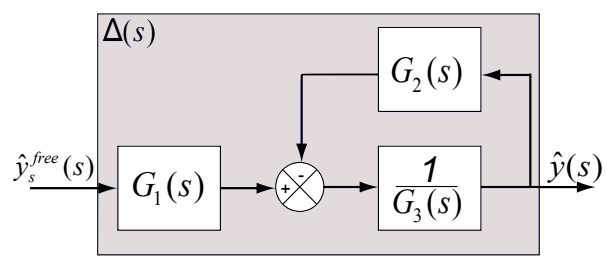

Fig. 5: Dynamics observer with inverse multiplicative structure.

From Fig. 5, we have:

$$
\frac{\hat{y}(s)}{\hat{y}_{s}^{\text {free }}(s)}=\frac{G_{1}(s)}{G_{2}(s)+G_{3}(s)}
$$

Using (Eq. 21) and (Eq. 22p, we yield the relation between complete estimate information $\hat{y}(s)$ and input control $U$ :

$\frac{\hat{y}(s)}{U(s)}=\left(\frac{C_{r}}{\alpha}-\frac{1}{\frac{\alpha}{R_{f p}} s}-\frac{k_{D A}}{\alpha\left(1+\tau_{D A} s\right)}-\frac{1}{\alpha} H\right)\left(\frac{G_{1}}{G_{2}+G_{3}}\right)$

Using the first equation in (Eq. 16) and (Eq. 23), we have:

$$
\frac{G_{1}(s)}{G_{2}(s)+G_{3}(s)}=\frac{d_{p} D(s)}{\left(\frac{C_{r}}{\alpha}-\frac{1}{\frac{\alpha}{R_{f p}} s}-\frac{k_{D A}}{\alpha\left(1+\tau_{D A} s\right)}-\frac{1}{\alpha} H(s)\right)}
$$

We can therefore define the observer gains as follows:

$$
\left\{\begin{array}{l}
G_{1}(s)=d_{p} D(s) \\
G_{2}(s)=-\frac{1}{\frac{\alpha}{R_{f p}} s}-\frac{k_{D A}}{\alpha\left(1+\tau_{D A} s\right)}-\frac{1}{\alpha} H(s) \\
G_{3}(s)=\frac{C_{r}}{\alpha}
\end{array}\right.
$$

As we can remark, there is no need of dynamics inversion in the observer gains. The only gain to be inverted is $G_{3}$ (see Fig. 5p and this is a non-null constant $\left(G_{3}=\frac{C_{r}}{\alpha} \neq 0\right)$ and is always invertible. Also, there is no additional computation or algorithm (such as least-square, etc.) required for the gains. As soon as the electrical and piezoelectric models are known and identified, the observer is directly obtained.

\section{Governing equations and block schemes of the complete observer}

Gathering the static force and displacement observer in (Eq. 5), the dynamics observer in (Eq. 22) and the observer gains in (Eq. 25), the governing equations of the complete observer are:

$$
\left\{\begin{array}{l}
\hat{F}_{s}(t)= \\
\frac{1}{\beta}\left(\left(C_{r} U(t)-C U_{o}(t)\right)-F_{c r}(t)-F_{h y s}(t)-\frac{1}{R_{f p}} \int_{0}^{t} U d t\right) \\
\hat{y}_{s}^{\text {free }}(t)= \\
\frac{1}{\alpha}\left(\left(C_{r} U(t)-C U_{o}(t)\right)-Q_{D A}(t, U)-\frac{1}{R_{f p}} \int_{0}^{t} U d t\right) \\
\hat{y}(s)=\left(\frac{G_{1}(s)}{G_{2}(s)+G_{3}(s)}\right) \hat{y}_{s}^{\text {free }}(s)-s_{p} \hat{F}_{s}(s)
\end{array}\right.
$$

with (according to (Eq. 3), (Eq. 6) and (Eq. 25)):

$$
\left\{\begin{array}{l}
F_{c r}(s)=F_{t f c r}(s) U(s) \\
F_{h y s}(s)=\Gamma(U) \\
Q_{D A}(s)=\frac{k_{D A}}{\left(1+\tau_{D A} s\right)} U(s)=Q_{t f D A}(s) U(s) \\
G_{1}(s)=d_{p} D(s) \\
G_{2}(s)=-\frac{1}{\frac{\alpha}{R_{f p}} s}-\frac{k_{D A}}{\alpha\left(1+\tau_{D A} s\right)}-\frac{1}{\alpha} H(s) \\
G_{3}(s)=\frac{C_{r}}{\alpha}
\end{array}\right.
$$

Fig. 6 presents the block diagram of the complete observer defined by (Eq. 26).

In the proposed observer (Eq. 26, we choose the hysteresis operator $\Gamma(U)$ to be a Prandtl-Ishlinskii operator. The main advantage of this hysteresis operator are: i) its high accuracy, and ii) the fact that it is more suitable for real-time implementation.. The Prandtl-Ishlinskii hysteresis operator is based on the superposition of several elementary hysteresis called backlash or play-operator defined by:

$$
\left\{\begin{array}{l}
y(t)=\max \left\{u(t)-r, \min \left\{u(t)+r, y\left(t-T_{s}\right)\right\}\right\} \\
y(0)=y_{0}
\end{array}\right.
$$

where $r$ is the threshold.

A more complex hysteresis based on $n_{h}$ number of backlashes is therefore defined as:

$\left\{\begin{array}{l}y(t)=\sum_{i=1}^{n_{h}} w_{i} \cdot \max \left\{U(t)-r_{i}, \min \left\{U(t)+r_{i}, y_{i}\left(t-T_{s}\right)\right\}\right\} \\ y(0)=y_{0}\end{array}\right.$

where $w_{i}, r_{i}$ and $y_{i}$ are the weighting and the threshold parameters and the output of the $i^{t h}$ backlash, respectively. 


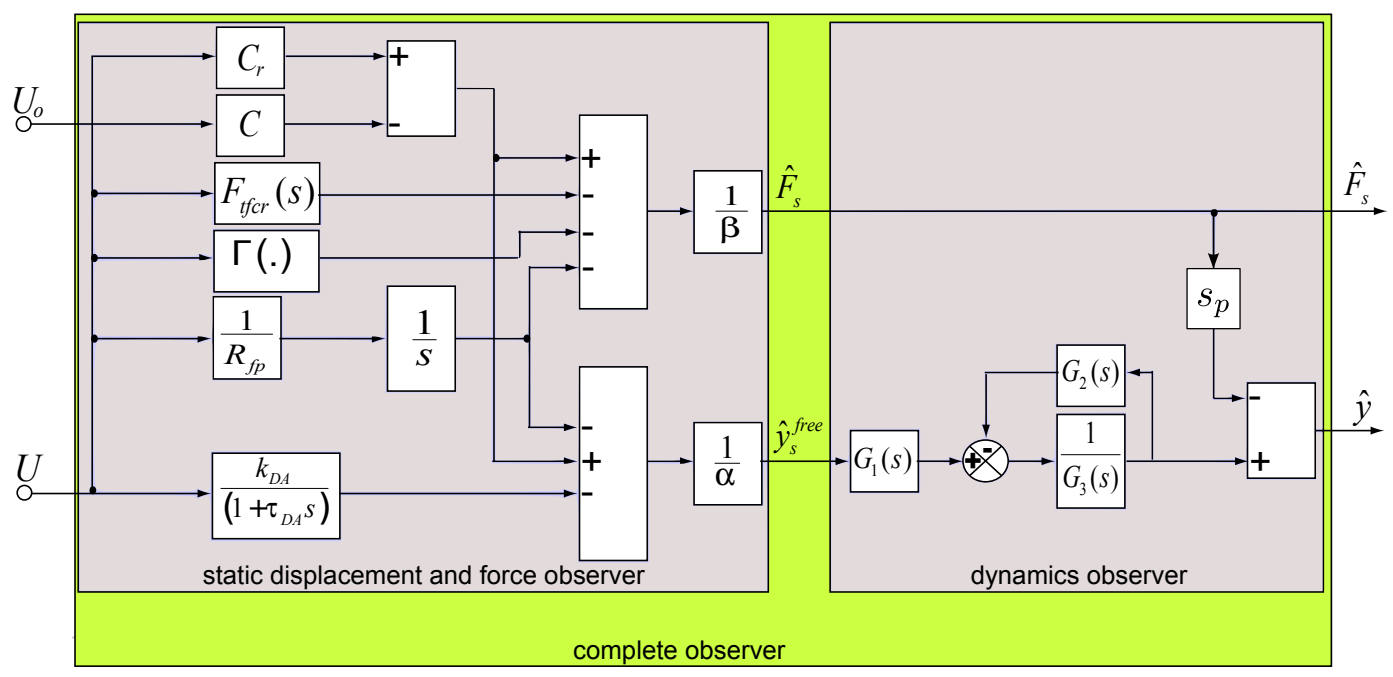

Fig. 6: Block diagram of the complete observer.

The creep operator $F_{t f c r}(s)$ in the observer is a transfer function (linear operator):

$$
F_{t f c r}(s)=\frac{\sum_{k=0}^{m} b_{k} s^{k}}{\sum_{l=0}^{n} a_{l} s^{l}}
$$

where $b_{k}$ and $a_{l}$ are the parameters to be identified and $m$ and $n(m \leq n)$ are the degrees of the polynomials. The operator's order is equal to $n$.

\section{E. Experimental results and setup}

The complete self-sensing was implemented and tested. The setup used, presented in Fig. 7, is composed of:

- a unimorph piezoelectric cantilever with dimensions of $15 \mathrm{~mm} \times 2 \mathrm{~mm} \times 0.3 \mathrm{~mm}$, where the thickness of the piezoelectric layer (lead zirconate titanate - PZT-5H) and the thickness of the passive layer (Nickel) are $0.2 \mathrm{~mm}$ and $0.1 \mathrm{~mm}$ respectively,

- a dSPACE-board (DS1103) and a computer material for the data acquisition, observer implementation and control signal. MATLAB-SimULinK is the software used for that. Based on the dynamics of the actuator, the sampling time is set equal to $T_{s}=50 \mu \mathrm{s}$.

- a displacement optical sensor (LC-2420 from Keyence, [33|) to measure the deflection (displacement) at the tip of the actuator. This sensor is set to have a resolution of some tens of nanometers and an accuracy of $100 \mathrm{~nm}$,

- a force sensor (FT-S270 from femtotools [34]) to measure the force applied by the actuator at its tip. The sensor has a range of $\pm 2 m N$, a sensitivity of $1 \frac{\mu N}{m V}$ and a resolution of $0.4 \mu N$.

- a custom-made electronic circuit based on Fig. 2-b. The used op-amp (OPA111) features very low bias currents $(1 p A)$ and a low noise,

- and a high voltage (HV) amplifier with differential output (KH7602M from Krohn-Hite) used to amplify the input voltage $U$ from the dSPACE-/computer. This amplifier can provide up to $\pm 200 \mathrm{~V}$ at its output.
It is noticed that the displacement and the force sensors are used here to capture the real displacement $y$ and the real force $F$ in order to compare them with the estimate $\hat{y}$ and $\hat{F}$ for the validation of the proposed approach. They are also used to identify some model parameters such as $d_{p} D(s)$.

The tip of the piezoelectric cantilevered actuator is positioned between the optical sensor head and the probe of the force sensor. The force sensor is mounted on a XYZ mechanical table which will bring it on close vicinity to the actuator's tip. Force may be applied either externally by adjusting the sensor position (e.g. pushing it against the cantilevered actuator), or through the cantilever subject to an applied voltage, which will solely enter in contact with the sensor, thus simulating a micromanipulation procedure. This second method permits to have a very high dynamics force, simulating a step micromanipulation force when applying a step voltage to the actuator entering in contact with an object. This paper presents the estimation of the final value of this step force, additionally to the bending of the actuator.

To identify the hysteresis $\Gamma(U)$, we choose a number of backlashes $n_{h}=15$. Then the parameters have been identified following the procedure proposed in [17]. The creep operator was identified based also on the procedure in [17].

The remaining parameters in (Eq. 26) are identified following the procedures in [25] [26]. The electrical elements are: $C=47 n F$ and $C_{r}=8.2 n F$. Finally for the given actuator, we identified and calculated $k_{D A}=-0.028 \mu \mathrm{m} / V, \tau_{D A}=60 \mathrm{~s}$, $\alpha=273 \mathrm{mV} / \mu \mathrm{m}, \beta=1.03 \mathrm{nC} / \mathrm{mN}$ and $R_{f p}=0.435 T \Omega$. The piezoelectric compliance $s_{p}$ has been identified by using the above mentionned external force sensor and displacement optical sensor. The force sensor is used to push the tip of the piezoelectric cantilever until a constant force $F$ is obtained and the resulting steady-state displacement is measured by the optical sensor. Different values of force ranging between $0 \mathrm{mN}$ and $2 m N$ were used. The results are given in Fig. 8 where we can notice the linearity of the force-displacement relation. From experimental data shown in the figure, a linear regression allows us to identify an average of $s_{p}=1.2858 \mu \mathrm{m} / \mathrm{mN}$ with a standard deviation of $0.08867 \mu \mathrm{m} / \mathrm{mN}$. 


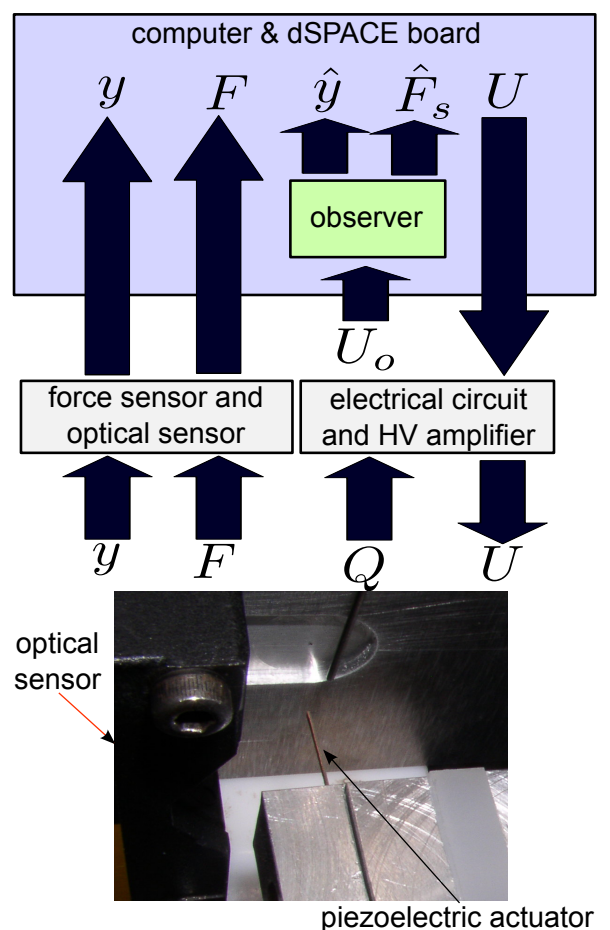

Fig. 7: Setup used for the experimental tests.

The identification of $d_{p}$ and $D(s)$ is performed by applying a step voltage $U$ to the actuator without force at the tip and then by capturing the output $y$ thanks to the optical sensor. After applying an ARMAX (Auto Regressive model with eXternal input) method to the captured data with MATLAB [38], we obtain:

$$
\left\{\begin{array}{l}
d p=0.690 \frac{\mu m}{V} \\
D(s)=5.752 \times 10^{-3} \times \\
\frac{\left(s+3.06 \times 10^{4}\right)\left(s^{2}-1.95 \times 10^{4} s+3.07 \times 10^{8}\right)}{(s+3976)\left(s^{2}+54.37 s+1.36 \times 10^{7}\right)}
\end{array}\right.
$$

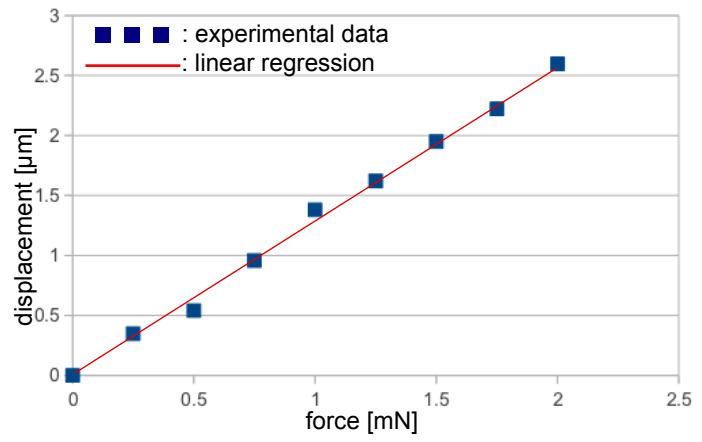

Fig. 8: Steady-state force-displacement characterization used to identify the piezoelectric compliance $s_{p}$.

At the same time, the output $U_{o}$ was captured allowing the identification of $H(s)$ :

$$
H(s)=\frac{-0.158\left(s+5.9 \times 10^{4}\right)(s+236)(s+13.7)}{\left(s+5.5 \times 10^{4}\right)(s+224)(s+12.9)}
$$

The complete observer with the identified parameters has been implemented. The first experiment consists in comparing the quasi-static displacement self-sensing presented in section. II-B with the proposed complete displacement selfsensing in free condition $F=0$. In such a way, we can observe the efficiency of the complete self-sensing to track high frequency or high rate evolution of the displacement. The experiment consists in applying a step input voltage $U=20 \mathrm{~V}$ to the actuator and to report the resulting real displacement $y$ thanks to the optical sensor. At the same time, the estimate displacement from the quasi-static displacement self-sensing and from the proposed complete displacement self-sensing are also reported. Then, transient parts of the displacement step response are observed. The observation is performed within only $200 \mathrm{~ms}$ because of the low settling time of the piezoelectric cantilever. Fig. 9 present the results. As we can see, the quasi-static self-sensing does not estimate the transient part of the real displacement (Fig. 9 a) and only estimates the steady-state. In contrary, the complete self-sensing captures both transient part and the steady-state value (Fig. 9 -b).

The next experiment consists in applying a series of force at the tip of the piezoelectric actuator thanks to the probe of the force sensor and thanks to a manual positioning table used to move the sensor towards the actuator. The reaction force of the actuator which is $F$ is the force to be observed/estimated by the self-sensing. At the same time, the displacement $y$ (deflection of the actuator) generated by the force is also estimated by the self-sensing. The aim of this experiment is to validate and demonstrate the efficiency of the proposed self-sensing to estimate both displacement and force. As the force is estimated in a quasti-static way, the measurement here is performed during several tens of seconds. Fig. 10 picture the results. From these figures, we remark that the estimate $\hat{F}$ derived by the sefl-sensing well captures the real force $F$ measured by the sensor (Fig. 10-a). Concerning the displacement, the estimate $\hat{y}$ captures enough the real displacement $y$ despite the slight inaccuracy of $\cong 200 \mathrm{~nm}$ for $3.1 \mu \mathrm{m}$ of range. This inaccuracy is acceptable for the considered applications since it is very negligible (much less than the micron). Comparing the results in Fig. 9 and in Fig. 10, we notice that the accuracy of the proposed self-sensing is much more better when the force $F$ is zero. The inaccuracy obtained when $F$ is non-zero can be reduced by using a more precise model of the piezoelectric compliance $s_{p}$. In this paper, we have proposed a linear model (see Fig. 8) but a high degree polynomial could be used in order to reduce this average error (standard deviation) and consequently to reduce the error of estimation of the force. It is worth to notice that the force and the displacement at the steady-state, pictured in Fig. 10-a and $b$ respectively, are not constant. In fact this is due to the creep phenomenon that typifies piezoelectric ceramics based actuators. Such a drift is observed at low frequencies or when a constant input voltage is applied. Our experiments clearly show that the self-sensing can efficiently track the appearance of this creep. To summarize, the characterization demonstrates that the proposed self-sensing well estimates both the transient part (high frequency) and the steady-state (low frequency) of the displacement of the piezoelectric actuator. In the steadystate, we see that the error of displacement estimation is less than $1 \%$. Concerning the force estimation, which is in the steady-state, the accuracy is slightly worse than that of 
the displacement: $6 \%$ of error over the period of observation $(100 s)$ and for an amplitude of $(3 m N)$. Despite this error, this is compatible with microassembly or micromanipulation tasks where the displacement is feedback controlled and the force is only displayed.

IV. APPLICATION TO A ROBUST FEEDBACK CONTROL OF THE DISPLACEMENT

In this part, we apply the proposed complete self-sensing method in a feedback control: the estimate displacement from the self-sensing will be used as feedback signal, the estimate force being displayed. We use the the standard $H_{\infty}$ technique to calculate the controller. Such technique has already demonstrated its efficiency to improve piezoelectric actuators performances in previous works [7]. But, relative to these previous works, the measurement for feedback in this paper is from self-sensing technique, not from an external sensor.

\section{A. The system to be controlled}

Fig. 11 presents the system to be controlled. In this, the real displacement $y$, which is unavailable for measurement, is estimated by the previous self-sensing. This estimate $\hat{y}$ is used for feedback. In parallel to the control of the displacement, the force $F$ applied by the actuator to the environment (manipulated object) is estimated by the same self-sensing (cf. previous section). Therefore, during the displacement control, the manipulation force can be displayed in real time. It is worth to notice that the optical sensor is still used to measure the real displacement $y$ and to compare the latter with the estimate during the control, and therefore for the validation.

\section{B. Sepcifications for the closed-loop}

To design the controller, we propose the following specifications. They are in accordance with the requirements in micropositioning tasks during micromanipulation and microassembly.

Tracking performances - The overshoot, which was initially $64 \%$ (see the step response of the system in Fig. 97, should be cancelled. The statical error should be negligible. Finally the settling time should be less than $100 \mathrm{~ms}$.

Limitation of the input voltage $U$ - In order to avoid the destruction of the piezoelectric actuator by eventual overvoltage, the input control $U$ should be limited. We choose a maximal ratio of $\frac{U_{\max }}{y_{\max }}=\frac{100}{20}\left[\frac{\mathrm{V}}{\mu m}\right]=5\left[\frac{\mathrm{V}}{\mu \mathrm{m}}\right]$ where $y_{\max }$ corresponds to the maximal range of use.

\section{C. $H_{\infty}$ controller synthesis}

One of the main advantages of the $H_{\infty}$ approach is the explicit consideration of the specifications. These specifications are transcribed into weighting functions during the synthesis. Consider in Fig. 12 a the closed-loop scheme for the controller design, where the weighting $W_{1}(s)$ and $W_{2}(s)$ are used to include the specified tracking performances and the specified voltage limitation respectively. In the figure:

- $y_{r}$ is the reference displacement,

- $\varepsilon=y_{r}-y$ is the error,

- $e_{1}$ and $e_{2}$ are the weighted outputs used for the synthesis,

- $G(s)$ is the system to be controlled, with $G(s)=d_{p} D(s)$,

- and $K(s)$ is the controller to be synthesized.

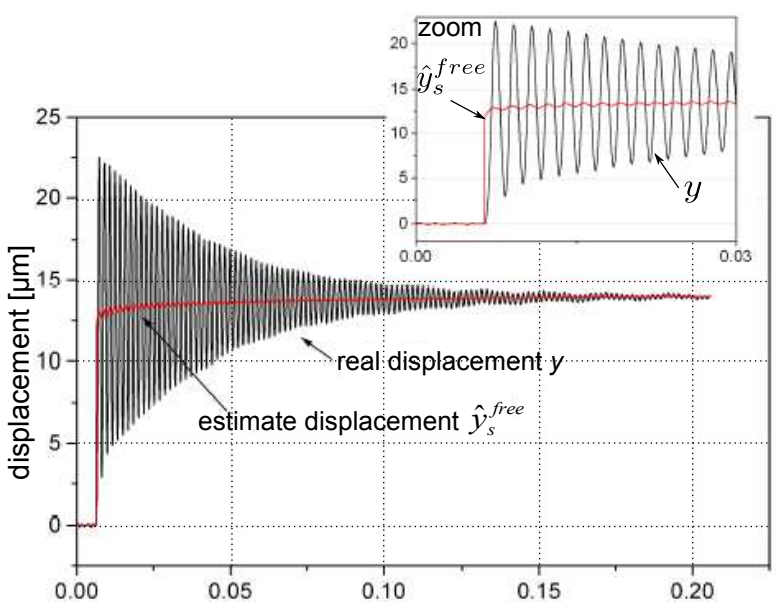

(a): obtained with the quasi-static displacement self-sensing

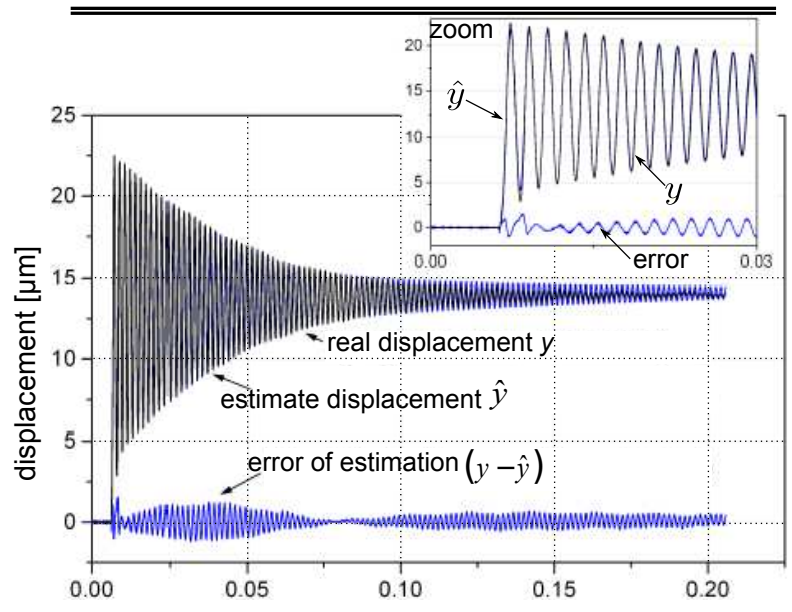

(b): obtained with the complete displacement self-sensing

Fig. 9: Experimental results. (a): displacement estimation (with force null) using the quasi-static self-sensing in [25] [26]. (b) results using the proposed complete displacement self-sensing.

The standard scheme corresponding to Fig. 12-a is pictured in Fig. 12 b. This is used for the standard $H_{\infty}$ synthesis. In this, $P(s)$ is called augmented system since it contains the system $G(s)$ augmented by the weightings $W_{1}(s)$ and $W_{2}(s)$. The exogenous input is the reference $y_{r}$ and the output to be controlled are the weighted signals $e_{1}$ and $e_{2}$. The standard $H_{\infty}$ problem consists in finding an optimal value $\gamma>0$ and a controller $K(s)$ stabilizing the closed-loop in Fig. 12-b (the standard scheme) and guaranteeing the following inequality [35:

$$
\left\|F_{l}(P(s), K(s))\right\|_{\infty}<\gamma
$$

where $F_{l}(P(s), K(s))$ is the lower linear fractionar transformation (LFT) between $P(s)$ and $K(s)$ and is defined by:

$$
\left(\begin{array}{c}
e_{1} \\
e_{2}
\end{array}\right)=F_{l}(P(s), K(s)) \cdot y_{r}
$$

From Fig. 12,a, we have:

$$
e_{1}=W_{1} S y_{r} \quad ; \quad e_{2}=W_{2} K S y_{r}
$$

where $S=(1+K G)^{-1}$ is the sensitivity function. Using (Eq. 34) and (Eq. 35), we have the LFT of the standard 
scheme:

$$
F_{l}(P(s), K(s))=\left(\begin{array}{c}
W_{1} S \\
W_{2} K S
\end{array}\right)
$$

Using the condition of the $H_{\infty}$ standard in (Ineq. 33) and the LFT in (Eq. 36), we infer the more detailed conditions:

$$
\left\|W_{1} S\right\|_{\infty}<\gamma \quad ;\left\|W_{2} K S\right\|_{\infty}<\gamma
$$

which is also respected if we have

$$
\left|W_{1}\right|<\frac{\gamma}{\left|W_{1}\right|} \quad ; \quad|K S|<\frac{\gamma}{\left|W_{2}\right|}
$$
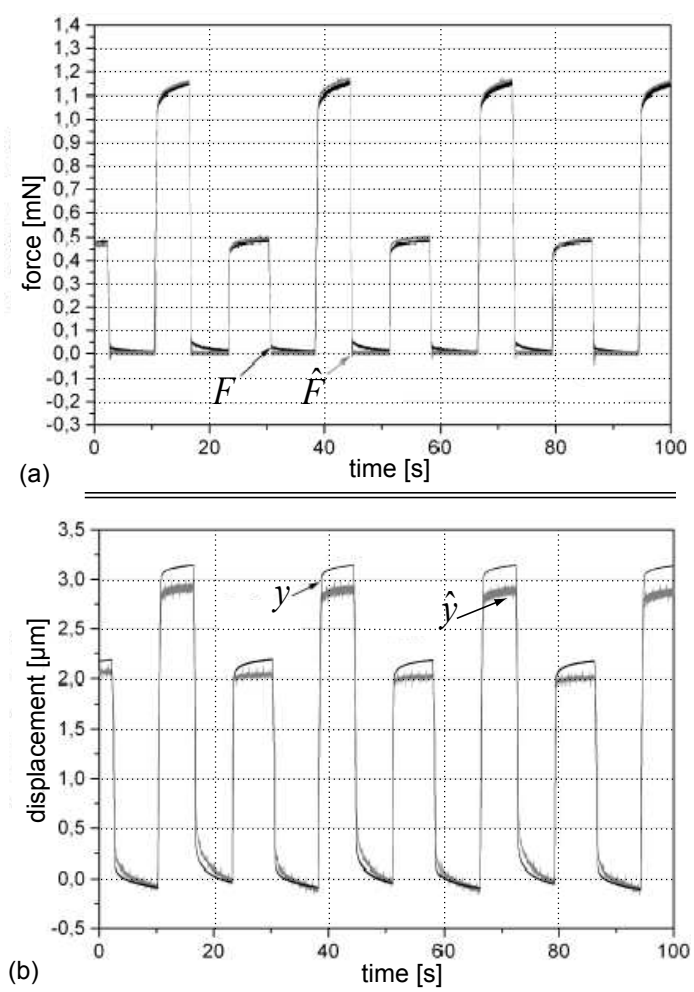

Fig. 10: Experimental results. (a): force $F$ measured by an external force sensor and estimate force $\hat{F}$ from the selfsensing. (b): displacement $y$ measured by an external optical sensor and estimate displacement $\hat{y}$ from the self-sensing.

To solve the problem (Ineq. 38) (or (Ineq. 37p), we use the Glover-Doyle algorithm which is based on the Riccati equations [36] 37].

\section{Computation of the controller}

The weighting functions were chosen according to the specifications in Section. IV-B We choose the following set:

$$
W_{1}(s)=\frac{0.03 s+1}{s+0.001} \quad ; \quad W_{2}=\frac{2 \times 10^{-5} s+1}{6.25 \times 10^{-5} s+5}
$$

After computation, we obtain a controller of order 5 :

$$
\left\{\begin{array}{l}
K(s)= \\
\frac{163\left(\mathrm{~s}+8 \times 10^{4}\right)(\mathrm{s}+3876)\left(\mathrm{s}^{2}+54 \mathrm{~s}+1.36 \times 10^{7}\right)}{\left(\mathrm{s}+5 \times 10^{4}\right)(\mathrm{s}+4137)(\mathrm{s}+0.001)\left(\mathrm{s}^{2}+519 \mathrm{~s}+1.4 \times 10^{7}\right)} \\
\gamma_{\text {optimal }}=0.2
\end{array}\right.
$$

$\gamma$ being less than 1 indicates that the controller will ensure the performances described in the specifications.

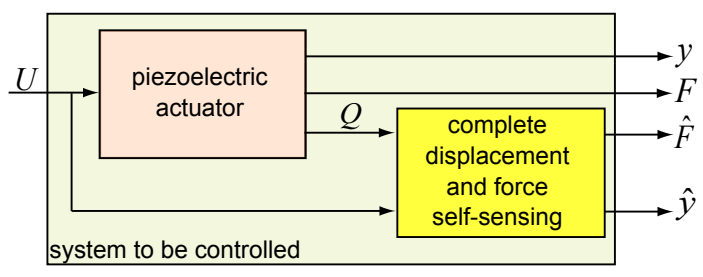

Fig. 11: The system to be controlled.

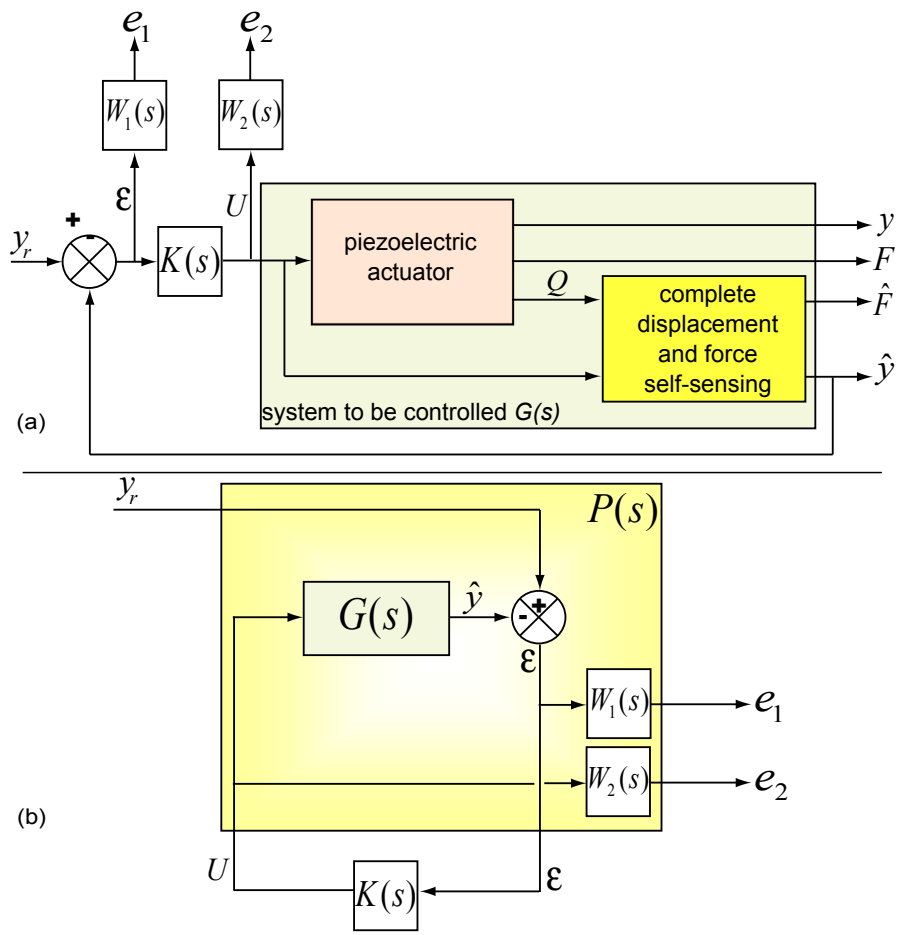

Fig. 12: (a): the closed-loop scheme augmented with the weightings. (b): the corresponding standard scheme.

\section{E. Experimental results}

The controller $K(s)$ in (Ineq. 40 has been implemented in the Matlab-SimulinK software. The first experiment consists in applying a series of steps for the reference input $y_{r}$ to the closed-loop system. No force sensor (or object) is in contact with the actuator in this first experiment such that $F=0$. The aim is to just validate the displacement tracking performances. Both the estimate displacement $\hat{y}$ that was used for the feedback and the real displacement $y$ that is measured thanks to the optical sensor are captured. Fig. 13 pictures the results. As we can see, the estimate displacement $\hat{y}$ well coincides with the real displacement $y$. On the other hand, they well tracks the input reference $y_{r}$. The specifications are particularly respected: there is no overshoot, the statical error tends towards zero and the settling time is less than $100 \mathrm{~ms}$.

In the second experiment, we apply arbitrary levels of $7^{7}$ ) reference input, but still with $F=0$. The objective is to evaluate the input voltage $U$ and the ability of the complete self-sensing to work in long term measurement (over a period of $200 \mathrm{~s}$ ) of displacement signal and with high voltage. Fig. 14 a pictures the reference displacement $y_{r e f}$, the measured displacement $y$ from the external optical sensor and the estimate 
displacement $\hat{y}$ from the self-sensing. Results shown in these figures demonstrate the efficiency of the control techniques using the self-sensing, where it can be verified that the output of the piezoelectric actuator well tracks the reference input $y_{\text {ref }}$. Fig. 14.b shows the high input voltage $U$ (up to $100 \mathrm{~V}$ ) applied by the controller $K(s)$ to the actuator. Fig. 14 c shows a zoom on the displacement response at time between $168 \mathrm{~s}$ and $187 \mathrm{~s}$ where the reference input displacement is $y_{r}=0 \mu \mathrm{m}$. From this latter figure, we observe that the signal obtained from the self-sensing technique is less noisy than that provided by the optical sensor. Although the reference $y_{r}$ is null and the output $y$ (and its estimate $\hat{y}$ ) is null, we remark from Fig. 14. c and Fig. 14 b that the corresponding driving voltage $U$ is non-null (about $-10 \mathrm{~V}$ ). This is due to the fact that this driving voltage is compensating for the nonlinearities (hysteresis and creep) of the piezoelectric actuator which demonstrates the efficiency of the designed controller.

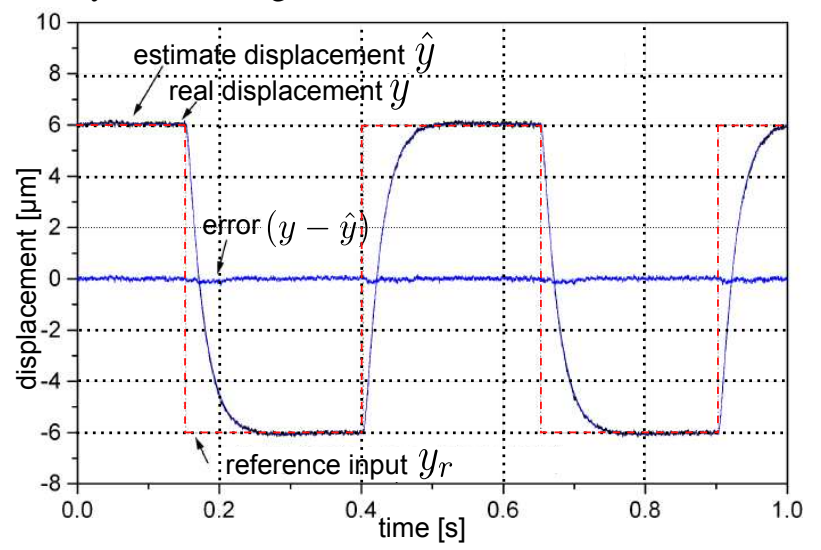

Fig. 13: Step response of the closed-loop system with $F=0$.

The last experiment consists in performing a reference tracking (tracking of $y_{r}$ ) when the actuator is in contact with the force sensor $(F \neq 0)$. During the experiment, the reference displacement $y_{r}$ is modified slightly in order to avoid brusque evolution that may destroy the force sensor. Fig. 15 picture the results. In Fig. 15 -a where the displacement $\hat{y}$ is shown, the values are shifted of $50 \mu \mathrm{m}$. In Fig. 15.b where the real force $F$ is shown, the values are shifted of $-0.3 m N$. The noises arrowed on the curve are due to a slight sliding between force sensor's probe and the actuator. Finally in Fig. 15 -c where the estimate force $\hat{F}$ is shown, the values are shifted of about $1 m N$. As we can see in the two latter figures, the estimate force well estimates the real force, in particular when the contact is reached. When the contact is off, there is a slight drift of the estimate force (Fig. 15 - c). This drift causes a shift of the estimate force relative to the real force during contact. This drift, which can be positive or negative, is due to the sensitivity of the self-sensing to the environmental conditions (temperature variation,...) but also in part due to the model of the compliance $s_{p}$. This drift can be minimized by utilizing a controlled environment for the experimental setup. Another approach consists in employing an environment-dependent self-sensing technique. In this, the parameters of the selfsensing are dependent on the evolution of the temperature. Finally, a more precise model of the compliance $s_{p}$ could also increase the accuracy of the force estimation.
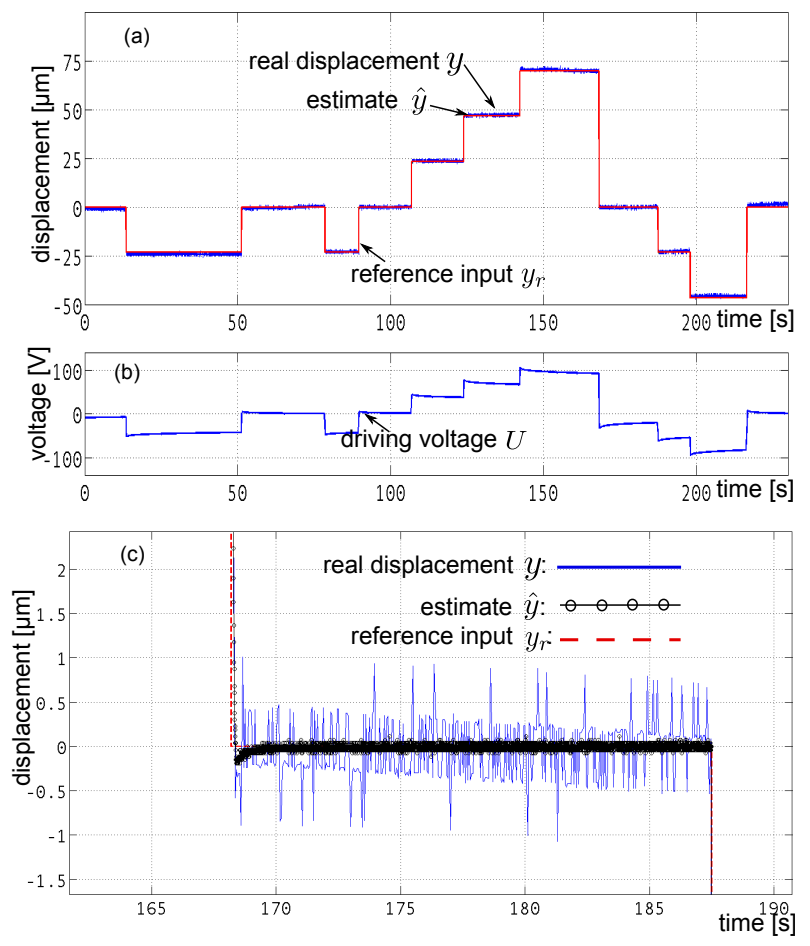

Fig. 14: Long term experiment with $F=0$ and with high input voltage $U$. (a): the reference displacement $y_{r e f}$ and the output displacement measured by the optical sensor $(y)$ and estimated $(\hat{y})$. (b): the driving voltage $U$. (c): a zoom on the displacement when the reference input $y_{r}=0 \mu \mathrm{m}$.

\section{CONClusion}

This paper has presented the development of a self-sensing technique, called complete self-sensing, that is able to estimate the force and the displacement in a piezoelectric actuators dedicated to micropositioning tasks. While the force is estimated at its steady-state (static), the displacement is estimated at both its transient part (dynamics) and its steady-state. The advantage is that it is possible to develop a feedback displacement control and at the same time to display the force applied by the actuator. Another advantage relative to existing self-sensing techniques is that the proposed approach can capture the two signals for long term (several hundred of seconds). The experiments carried on a feedback control have demonstrated these advantages.

The accuracy of self-sensing techniques depends on the accuracy of the identified parameters. If they are badly identified, the self-sensing will provide inaccurate estimate of the displacement or force. These parameters may also change over the time, for instance due to thermal variation. A perspective work at FEMTO-ST consists in developing self-adjusted selfsensing techniques where the parameters are automatically (in real-time) tuned according to the temperature changes.

\section{ACKNOWLEDGMENT}

This work is supported by the national ANR-Emergence MYMESYS-project (ANR-11-EMMA-006: High Performances Embedded Measurement Systems for multiDegrees of Freedom Microsystems) and partially supported by the Romanian project UEFISCDI PN-II-RU-TE-2011-3-0299 "Ad- 
vanced devices for micro and nanoscale manipulation and characterization (ADMAN).
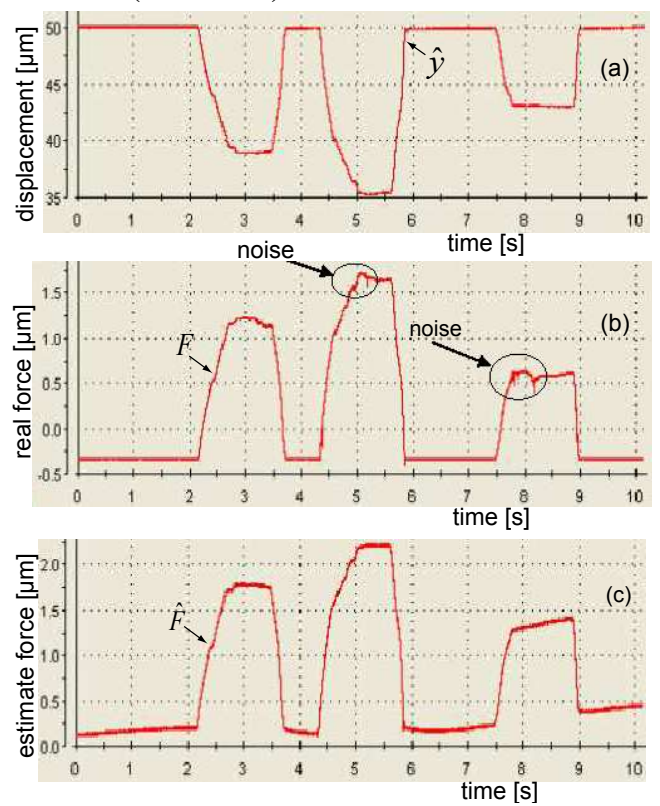

Fig. 15: Experimental result on tracking of a reference displacement $y_{r}$ with $F \neq 0$ (the actuator is in contact with an object). (a): the estimate displacement $\hat{y}$. (b): the measured (real) force $F$. (c): the estimate force from the self-sensing measurement technique.

\section{REFERENCES}

[1] A. Bergander, W. Driesen, T. Varidel, M. Meizoso and J. M. Breguet, "Mobile $\mathrm{cm} 3$-microrobots with tools for nanoscale imaging and micromanipulation", Mechatronics \& Robotics, pp.1041-1047, Aachen, Germany, Sept 2004.

[2] M. Rakotondrabe, Y. Haddab and P. Lutz, "Development, modelling and control of a micro/nano positioning 2DoF stick-slip device", IEEE/ASME Trans. on Mechatronics , Vol.14(6), pp:733-745, Dec 2009.

[3] G. Bining, C. F. Quate and Ch. Berger, "Atomic Force Microscope", APS Physical Review Letters (physics), Vol.56, pp.930-933, 1986.

[4] J. Agnus, J. M. Breguet, N. Chaillet, O. Cois, P. de Lit, A. Ferreira, P. Melchior, C. Pellet and J. Sabatier, "A smart microrobot on chip: design, identification and modeling", IEEE/ASME Intern. Conf. on Advanced Intelligent Mechatronics, Kobe Japan, pp.685-690, July 2003.

[5] M. Rakotondrabe and Ioan A. Ivan, "Development and Force/Position Control of a New Hybrid Thermo-Piezoelectric microGripper dedicated to automated pick-and-place tasks", IEEE Trans. Automation Science and Engineering, Vol.8(4), pp.824-834, Oct. 2011.

[6] K. K. Leang and S. Devasia, "Hysteresis, creep, and vibration compensation", IFAC Conference on Mechatronic Systems, pp.283-289, Berkeley CA USA, Dec. 2002.

[7] M. Rakotondrabe, Y. Haddab and P. Lutz, "Quadrilateral modeling and robust control of a nonlinear piezoelectric cantilever", IEEE Trans. on Control Systems Technology, Vol.17(3), pp.528-539, May 2009.

[8] S. Khadraoui, M. Rakotondrabe and P. Lutz, "Interval Modeling and Robust Control of Piezoelectric Microactuators", IEEE - Trans. on Control Systems Technology, Vol.20(2), pp.486-494, March 2012.

[9] C. Lee and S. Salapaka, 'Fast Robust Nanopositioning - A Linear Matrix Inequalities Based Optimal Control Approach', IEEE/ASME Trans. on Mechatronics, Vol.14(4), 414-422, Aug. 2009.

[10] A. Fleming and SORM Moheimani, "A grounded load charge amplifier for reducing hysteresis in piezoelectric tube scanners," Review of Scientific Instruments, Vol.76(7), 073707(1-5), July 2005.

[11] G. M. Clayton, S. Tien, A. J. Fleming, SOR. Moheimani, S. Devasia, "Inverse-feedforward of charge-controlled piezopositioners", Mechatronics, Vol.(5-6), page 273-281, June 2008.

[12] M. Rakotondrabe, "Bouc-Wen modeling and inverse multiplicative structure to compensate hysteresis nonlinearity in piezoelectric actuators", IEEE Trans. on Automation Science and Engineering, Vol.8(2), pp.428431, April 2011.
[13] S. Bashash and N. Jalili, "A Polynomial-Based Linear Mapping Strategy for Feedforward Compensation of Hysteresis in Piezoelectric Actuators", ASME Journal of Dynamic Systems, Measurement and Control, Vol.130(3), May 2008.

[14] K. Kyle Eddy, "Actuator bias prediction using lookup-table hysteresis modeling", US Patent-08/846545, February 1999.

[15] D. Croft, G. Shed and S. Devasia, "Creep, hysteresis and vibration compensation for piezoactuators: atomic force microscopy application", ASME Journal of Dynamic Systems, Measurement and Control, Vol.123(1), pp.35-43, Mars 2001.

[16] A. Dubra and J. Massa and C.1 Paterson, "Preisach classical and nonlinear modeling of hysteresis in piezoceramic deformable mirrors", Optics Express, Vol.13(22), pp.9062-9070, 2005.

[17] M. Rakotondrabe, C. Clévy and P. Lutz, "Complete open loop control of hysteretic, creeped and oscillating piezoelectric cantilevers", IEEE Trans. on Automation Science and Engin., Vol.7(3), pp:440-450, July 2009.

[18] W. T. Ang, P. K. Kholsa and C. N. Riviere, "Feedforward controller with inverse rate-dependent model for piezoelectric actuators in trajectorytracking applications", IEEE/ASME Trans. on Mechatronics, Vol.12(2), pp.134-142, April 2007.

[19] B. Mokaberi and A. A. G. Requicha, "Compensation of scanner creep and hysteresis for AFM nanomanipulation", IEEE Trans. on Automation Science and Engineering, Vol.5(2), pp.197-208, April 2008.

[20] S. Devasia, E. E. Eleftheriou, R. Moheimani, "A survey of control issues in nanopositioning", IEEE Trans. on Control System Technology, Vol.15, No15, pp.802-823, 2007.

[21] J.J. Dosch, D.J. Inman and E. Garcia, "A Self-Sensing Piezoelectric Actuator for Collocated Control", Journal of Intell. Mater. Syst. and Struct., vol 3, pp. 166-185, 1992.

[22] T. Takigami, K. Oshima, Y. Hayakawa and M. Ito, "Application of selfsensing actuator to control of a soft-handling gripper", Proc. to IEEE ICCA, pp. 902-906, Italy, 1998.

[23] Y. Cui, Self-Sensing, "Compounding Control of Piezoceramic MicroMotion Worktable Based on Integrator", Proc. to 6th World Congress on Intell. Cont. and Autom., China, 2006.

[24] A. S. Putra, H. Sunan, T. K. Kok, S.K. Panda and T. H. Lee, Self-sensing actuation with adaptive control in applications with switching trajectory, IEEE/ASME Trans. on Mechatronics, Vol.13(1), pp.104-110, 2008.

[25] A. Ivan, M. Rakotondrabe, P. Lutz and N. Chaillet, "Quasi-static displacement self-sensing method for cantilevered piezoelectric actuators", Review of Scient. Instr., Vol.80(6), 065102, June 2009.

[26] A. Ivan, M. Rakotondrabe, P. Lutz and N. Chaillet, "Current integration force and displacement self-sensing method for cantilevered piezoelectric actuators", Review of Scient. Instr., Vol.80(12), 2126103, Dec 2009.

[27] Y. Ishikiriyama and T. Morita, "Improvement of self-sensing piezoelectric actuator control using permittivity change detection", Journal of Advanced Mechanical Design, Systems, and Manufacturing,Vol.4, 2010.

[28] K. Kundert, "Modeling dielectric absorption in capacitors", The designer's guide community, pp.1-19, 2008.

[29] M. Rakotondrabe, A. Ivan, S. Khadraoui, C. Clévy, P. Lutz and N. Chaillet, "Dynamic displacement self-sensing and robust control of cantilevered piezoelectric actuators dedicated to microassembly tasks", IEEE/ASME Inter. Conf. on Intelligent Mechatronics, pp:557-562, Canada, July 2010.

[30] S. Devasia, "Should Model-based Inverse Inputs be used as Feedforward under Plant Uncertainty?" IEEE Trans. on Automatic Control, Vol. 47(11), pp. 1865-1871, Nov 2002.

[31] A. J. Fleming and KK. Leang, "Measurement and control for high-speed sub-atomic positioning in scanning probe microscopes", Workshop talk, "Signals measurement and estimation techniques issues in the micro/nanoworld", Anchorage, Alaska USA, May 2011.

[32] M. Rakotondrabe, C. Clévy, K. Rabenorosoa and K. Ncir, "Presentation, Force Estimation and Control of an Instrumented platform dedicated to Automated Micromanipulation Tasks", Int. Conf. on Automation Science and Engin., pp:722-727, Toronto Ontario Canada, August 2010.

[33] www.keyence.com

[34] www.femtotools.com

[35] G. J. Balas, J. C. Doyle, K. Glover, A. Packard and R. Smith, ' $\mu$-analysis and synthesis toolbox', The Mathworks User's Guide-3, 2001.

[36] K. Glover and J. C. Doyle, 'State-space formulae for all stabilizing controllers that satisfy an $H_{\infty}$-norm bound and relations to risk sensivity', Systems \& Control Letters, vol.11, pp.167-172, 1988.

[37] J. C. Doyle, K. Glover, P. K. Khargonekar and B. A. Francis, 'Statespace solutions to standard $H_{2}$ and $H_{\infty}$ control problems', IEEE Trans. on Automatic Control, AC 34 Nř8, pp.831-846, 1989.

[38] L. Ljung, "System identification toolbox", The Matlab user's guide. 